\title{
Network Slicing Resource Allocation and Monitoring over Multiple Clouds and Networks
}

\author{
Ricardo Martínez ${ }^{1}$, Ricard Vilalta1, Ramon Casellas ${ }^{1}$, Raul Muñoz ${ }^{1}$, Li Fei ${ }^{2}$, Pengcheng Tang ${ }^{2}$, Victor López ${ }^{3}$ \\ ${ }^{1}$ CTTC, Spain. \\ ${ }^{2}$ Huawei, Shangai, China \\ ${ }^{3}$ Telefónica Global CTO, Spain.
}

\begin{abstract}
This demo presents an in-operation network slice resource allocator, which is able to consider networking and cloud infrastructure. Monitoring cloud and network resources allows enhancing the (re-)allocation of network slices, while accommodating novel slice requests.

OCIS codes: (060.4250) Networks; (060.4510) Optical communications
\end{abstract}

\begin{abstract}
1. Introduction
Within the 5G environment, one of the most appealing capabilities is the support of a wide range of services and use cases / business arising from different vertical industries (IoT, eHealth, Industry4.0, etc.). Each of these services / business cases impose their own set of requirements (e.g., security, latency, resiliency, and bandwidth). To deal with this challenge, the NGMN has proposed the concept of network slicing [1]. Briefly, a network slice (NS) instance is formed by a set of network functions and logical resources enabling the deployment of a complete logical network infrastructure capable to accommodate the requirements for a specific service.

The network function refers to the processing functions within the network, i.e., elements and devices (e.g., packet and optical switches, transponders, RAN antennas, etc.). This encompasses both those ones provided by physical and dedicated appliances and those ones virtualized exploiting the NFV concept. A slice controller has been proposed by [2] to allocate network slices on top of an ETSI NFV architecture. Slice Controller is the functional block that within the OSS is responsible for interacting with the NFV MANO to control slicing. Within a Slice Controller, one can identify two main functions: network slice management and mapping of NSs to NFV network services.

Network resources are also important to be considered in both the intra-datacenter (intra-DC) and inter-datacenter (inter-DC), when allocating resources. Intra-DC networking (typically using leaf-spine architectures) is not considered to be a constraint for network slice placement, although availability of PCI express pass-through might limit the network function placement. For inter-DC, typically bandwidth availability is the main service requirement to be dealt with, but also network latency and resiliency should also be taken into account. ETSI NFV has also investigated on this topic, by proposing a WAN Infrastructure Manager (WIM), where inter-DC network control is delegated [4]. In this demo, the authors present NetSlice Planner, a framework for researching on NS resource allocation algorithms. NetSlice is able to run during network operation phase.
\end{abstract}

\section{Joint SDN and NFV demonstration}

The proposed 5G slicing architecture [5] aims at providing multiple, highly flexible, end-to-end network and cloud infrastructure slices operated in parallel over the same physical infrastructure to fulfill vertical-specific requirements as well as mobile broadband services. A NS is composed of tailored virtualized network and cloud resources, as well as the required virtualized network services We present the validated 5G architecture that supports the dynamic deployment of $5 G$ network slices on top of an ETSI NFV architecture (see Fig 1.a). It is composed of five main building blocks namely, the virtualized infrastructure managers (VIMs), WIMs, NFVO, Slice Controller, and the demonstrated NetSlice Planner.

The VIM allows to configure multiple tenants in a DC site, and to instantiate the creation/ deletion of virtual machine (VM) instances, storage of disk images, and the management of the intra-DC network connectivity for each tenant. The VIM also provides current status for these resources. WIM acts as an inter-DC network controller, allowing interVIM connectivity. Typically, VIMs are interconnected using a software-defined metro network. The WIM acts as the metro SDN controller.

The NFVO is responsible for deploying network services. The VNFs run in VMs that can be located in the most appropriate distributed cloud infrastructure, and are interconnected with each other and with the service end-points. The NFVO requests to the VIMs/WIMs the provisioning of the connectivity between the VNFs, and to the VNF Managers the lifecycle management of the VNFs. As explained, the Slice Controller is responsible for mapping NS to NFV network services, while tackling NS lifecycle [2].

Finally, the NetSlice Planner is the responsible for in-operation resource allocation. Firstly, the Request handler is responsible for receiving the NS requests (which in our demo will be statistically generated, such as in Fig 1.b). Secondly, the NetSlice Placement runs the selected resource allocation algorithm and provides a mapping between 
the logical requested resources towards the underlying discovered resources, which are requested to the model-driven infrastructure database (third step). The resources have been discovered from the underlying configured set of VIMs/WIMs (ONOS and OpenStack in our demo). Fourthly, once the resources are computed/selected, the NS request and its resource allocation are forwarded to the Slice Controller, which will finally trigger the setup of the necessary network services on top of the allocated infrastructure.

Fig 1.c shows the NSFNet scenario that will be considered for the demonstration. Apart of network elements, several DCs have been introduced to provide NFVI capabilities. Fig 1.d shows the detailed view of each DC network and compute resources (based on a leaf-spine architecture). NS requests will be statistically generated, and NetSlice Planner will compute the resource placement according to the considered allocation algorithms. As a result, the NS blocking probability will be obtained for different sets of configured traffic loads.
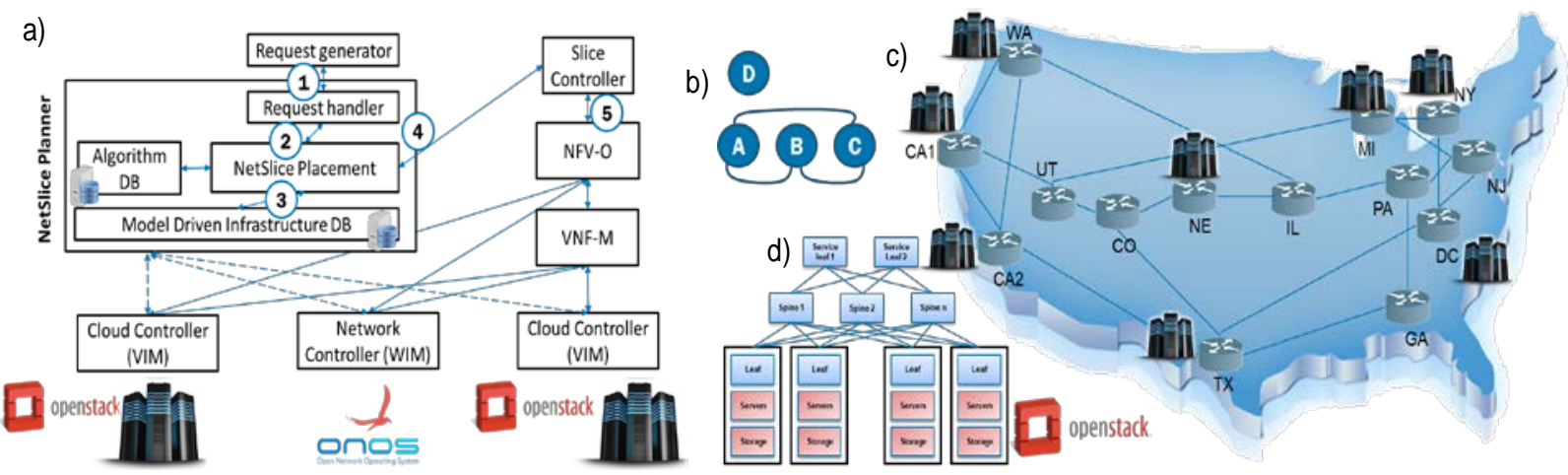

Figure 1. a) NetSlice Planner architecture and integration with ETSI NFV, b) Example of a network slice request; c) NSFNet Simulation scenario with interconnected DCs; and d) Zoom-in of intra-DC network architecture for simulation

\section{Innovation}

Several innovations are presented in this demonstration:

a) NetSlice Planner: Integration of a Network Slicing on-line planning tool with 3GPP and ETSI NFV architecture

b) Multi-VIM/WIM NFVI: Multi-site and multi-domain resources integration. VxLAN tunneling between VIMs.

c) Joint cloud and network resource allocation and monitoring: Common data models for resource status monitoring and optimized resource (re-)allocation.

\section{Relevance}

This demo is of interest of audiences typically addressing N2 and N3 committees. By attending this demo, the participants will gain knowledge on open source and standardization initiatives regarding control and management of transport networks, as well as inter-domain NFV scenarios.

\section{Acknowledgment}

The research leading to these results has received funding from EC H2020 5GPPP project 5GTANGO (761493) and MINECO project DESTELLO (TEC2015-69256-R).

\section{References}

[1]3GPP TR 28.801, Study on management and orchestration of network slicing for next generation network (Release 14 ), March 2017. [2]Bruno Chatras, Steve Tsang Kwong U, Nicolas Bihannic, NFV Enabling Network Slicing for 5G, ICIN, 2017.

[3]H. Dempo, et al. , Mapping ETSI-NFV onto Multi-Vendor, Multi-Domain Transport SDN, ETSI NFV PoC 42, March 2017.

[4]A. Mayoral, R. Vilalta, R. Muñoz, R. Casellas, R. Martínez, V. López, Cascading of tenant SDN and cloud controllers for 5G network slicing using Transport API and Openstack API , in Proceedings of International Conference on Optical Fiber Communications (OFC), 19-23 March 2017, Los Angeles (USA).

[5]A. Mayoral, R. Vilalta, R. Casellas, R. Martínez, R. Muñoz, Multi-tenant 5G Network Slicing Architecture with Dynamic Deployment of Virtualized Tenant Management and Orchestration (MANO) Instances , in Proceedings of 42nd European Conference and Exhibition on Optical Communications, 18-22 September 2016, Düsseldorf, Germany 